\title{
Cross-cultural adaptation and validation of the Romanian Knee disability and Osteoarthritis Outcome Score for Joint Replacement (KOOSJR)
}

\section{Sorin Florescu}

Universitatea de Medicina si Farmacie Victor Babes din Timisoara

\section{Dinu Vermesan}

Universitatea de Medicina si Farmacie Victor Babes din Timisoara

Horia Haragus ( $\sim$ horia.haragus@yahoo.com )

Universitatea de Medicina si Farmacie Victor Babes din Timisoara https://orcid.org/0000-0001-68013471

\section{Jenel M Patrascu Jr}

Universitatea de Medicina si Farmacie Victor Babes din Timisoara

\section{Bogdan Timar}

Universitatea de Medicina si Farmacie Victor Babes din Timisoara

\section{Adrian Todor}

Universitatea de Medicina si Farmacie luliu Hatieganu Facultatea de Medicina

\section{Research article}

Keywords: knee joint, cartilage, osteoarthritis, arthroplasty, Knee disability and Osteoarthritis Outcome Score for joint replacement, International Knee Documentation Committee, patient reported outcome measures

Posted Date: December 3rd, 2019

DOI: https://doi.org/10.21203/rs.2.18127/v1

License: (c) (i) This work is licensed under a Creative Commons Attribution 4.0 International License. Read Full License 


\section{Abstract}

Aim: To perform validation of the Romanian Knee disability and Osteoarthritis Outcome Score for Joint Replacement (KOOSJR).

Method: 96 patients (101 knees) with advanced osteoarthritis (OA) scheduled for total knee replacement completed Romanian translations of KOOSJR and IKDC (International Knee Documentation Committee subjective knee form) and Euroqol EQ-5D-5L, and the treating physician completed the original knee society score (KSS).

Results: Average age was 66.4 (range 50-83) years and male to female ratio 1:3.76. There was moderate correlation between the test-retest (average 4 days) KOOSJR $(r=0.618, n=45)$ and IKDC $(r=-0.671, n=99)$, weak between KOOSJR and EQ-5D-5L Index $(r=-0.431, n=100)$ and VAS $(r=-0.364, n=99)$ and very weak to KSS score $(r=-0.133, n=98)$ and function $(r=-0.072, n=97)$ For the first KOOSJR, Cronbach's alpha was 0.816 and intraclass correlation coefficient (ICC) $0.816(95 \% \mathrm{Cl} 0.755-0.866)$ for average measures. For the retest, Cronbach's alpha was $0.841(95 \% \mathrm{Cl} 0.760-0.903)$ for averages.

Conclusion: The Romanian Knee disability and Osteoarthritis Outcome Score for Joint Replacement (KOOSJR) is a valid, reliable, consistent and reproducible clinical score for patients with OA requiring arthroplasty.

\section{Introduction}

Osteoarthritis (OA) represents the degeneration of synovial joints. The knee is the most frequently involved of the large joints and its pathogenesis is not fully understood. It is accepted that OA is a disease of the entire joint, affecting the cartilage, subchondral bone and synovial tissue [1]. The prevalence of knee pain and symptomatic $O A$ is increasing with a surge in knee arthroplasties [2].

Progression is slow and for end stages the current treatment is joint replacement. Implant survival is $95 \%$ at 10 years yet up to $20 \%$ of patients are reported to be dissatisfied. Patient-reported outcomes (PROs) are therefore an integral part of routine clinical evaluation as well as national arthroplasty registries [3]. It is recommended to use a generic tool for quality of life assessment in conjunction with a disease specific score. Knee injury and Osteoarthritis Outcome Score (KOOS) is one of the most commonly used for total knee replacement $[3,4]$. A much shorter version (KOOSJR) has been proposed specifically for use in knee joint replacement. It has similar psychometric properties and its use is supported by national arthroplasty registries [3-5].

We aimed to perform validation of the Romanian translated Knee disability and Osteoarthritis Outcome Score for Joint Replacement (KOOSJR) in patients with advanced knee OA requiring joint replacement. 


\section{Materials And Methods}

We included 96 patients (5 bilateral) with advanced knee OA scheduled for total joint replacement, from Oct 2017 until Feb 2019. Diagnosis was made using the ACR (American College of Rheumatology) or EULAR (European League Against Rheumatism) criteria for knee osteoarthritis. Indication for arthroplasty was based on clinical and radiographic criteria (Kellgren-Lawrence grades III-IV). Bilateral cases underwent staged surgery 6 months apart. The study was conducted in accordance with the Declaration of Helsinki and the protocol was approved by the Emergency clinical county hospital 'Pius Brinzeu' Timisoara 'Local ethics committee for scientific research'. All patients gave their informed consent for inclusion before they participated in the study.

The subjects completed the Romanian translations of KOOSJR and IKDC (International Knee Documentation Committee - subjective knee form) in the clinics during the preoperative evaluation $[5,6]$. The treating physician (orthopedic surgeon) then completed the two parts of the original (1989) version of the knee society score (KSS1 - part1 knee score and KSS2 - part 2 function) [7]. Euroqol EQ-5D-5L Index (converted using the UK tariff) and visual analogue scale (VAS) were used to determine the general health status [8].

The full KOOS has been previously translated into Romanian and is available on the developer's website, yet no validation study exists [9]. Equivalent questions (S6, P2, P3, P6, P9, A3 and A5) and instructions were retrieved from the full questionnaire. IKDC was translated and culturally adapted in accordance with ISPOR (International Society for Pharmacoeconomics and Outcomes Research) principles of good practice.

Convergent validity was tested using Spearmans's correlation coefficient between the tested scores. Reliability and internal consistency were determined using Cronbach's alpha coefficient and intraclass correlation coefficient (ICC, two-way mixed effects model) [10]. A subgroup of patients repeated the KOOSJR after an average of 4 days (range 2-7) for the test-retest reproducibility assessment using Spearmans's correlation. For all tests, higher values are associated with better results. Data were analyzed using SPSS v17 statistical software package (SPSS Inc, Chicago, IL, USA).

\section{Results}

One hundred valid sets were available for processing. Average age was 66.4 (range 50-83) years and male to female ratio $21: 79$ (1:3.76). 46 subjects repeated the KOOSJR after 4 days. There were no floor or ceiling effects for both KOOSJR scores (min 0 - max 28). Raw summed scores ranged from 7 to 26 for the first and 9-27 for the second.

Twelve out of 14 consecutive patients ( 2 declined) were interviewed and timed at the first completion of the KOOSJR. Ten required glasses to read the questionnaire. Ten patients completed the score in an 
average of 2 minute and 34 seconds and found it clear and straight forward. Four estimated that they could complete the questionnaire through mail and phone and even email or tablet with assistance from family members. There were 2 outliers, which required repeated assistance from the investigator and family members.

There was moderate correlation between the first and repeated administration of the KOOSJR $(r=0.618$, $n=45)$ and IKDC ( $r=-0.671, n=99)$, weak between KOOSJR and EQ-5D-5L Index $(r=-0.431, n=100)$ and VAS $(r=-0.364, n=99)$ and very weak to KSS score $(r=-0.133, n=98)$ and function $(r=-0.072, n=97)$ (see Table. 1 , Figures 1 and 2).

Table.1

Figure.1

Figure.2

Internal consistency was strong. For the first KOOSJR, Cronbach's alpha was 0.816 and intraclass correlation coefficient (ICC) 0.387 for single $(95 \% \mathrm{Cl} 0.305-0.480)$ and 0.816 for average $(95 \% \mathrm{Cl} 0.755$ 0.866) measures respectively. For the retest, Cronbach's alpha was 0.841 and intraclass correlation coefficient (ICC) 0.431 for single $(95 \% \mathrm{Cl} 0.311-0.569)$ and 0.841 for average $(95 \% \mathrm{Cl} 0.760-0.903)$ measures.

\section{Discussion}

The Romanian translation of the KOOSJR proved valid, reliable, consistent and reproducible in patients with end stage OA undergoing total knee replacement. The Cronbach's alpha and ICC were comparable to recently published literature regarding KOOS translations: Spanish Cronbach's $0.78-0.93$ and ICC 0.76 to 0.91; Finnish Cronbach's 0.79-0.96 and ICC 0.73-0.86; Chinese Cronbach's 0.76-0.97 and ICC 0.89-0.95 and Greek ICC 0.76-.89 [11-14]. A direct comparison to similar translations of KOOSJR is difficult since data is only available for the full KOOS. This later has 7 questions for symptoms, 9 for pain, 17 for activities of daily living, 5 for sports and 4 for quality of life for a total of 425 point Likert scale items. It is freely available, self-explanatory, comprehensive, widely used for knee injuries leading to arthritis or OA and also includes the proprietary WOMAC (Western Ontario and McMaster Universities Osteoarthritis Index) $[5,9]$. Nevertheless, for routine clinical use especially in elderly, shorter scores are favored for compliance and efficiency. The 7-question standardized KOOSJR was proven to be just as reliable in patients undergoing total knee replacement. Other shortened versions of the KOOS have been proposed: the 7 question KOOS-PS (physical activity) and recently the 12 question KOOS-12 [15]. A single question - the M-SANE asked patients to rate their native or prosthetic knee on a scale from 0 to 10 . It corelated strong to moderate to KOOSJR and PROMIS (Patient-Reported Outcomes Measurement Information 
System) physical component [16]. The PROMIS uses computer adaptive testing, where algorithms select the best questions from a larger database. It is aimed at offering a unified tool for use in different pathologies as well as integrate disease specific points to activities of daily living. Its responsiveness is comparable to KOOSJR and HOOSJR (Hip disability and Osteoarthritis Outcome Score for Joint Replacement) in patients undergoing total joint arthroplasty [17]. Our study has several limitations. Firstly, we did not use the entire KOOS and secondarily subtract the KOOSJR. We felt that the full score might have been rather cumbersome to use in current elderly population undergoing knee arthroplasty in Romania and the simplified KOOSJR was proven to offer comparable usefulness [3-5,15]. Furthermore, we did not test responsiveness, by including a timepoint test after surgery and the translated Romanian IKDC form, the strongest comparator for validity is currently undergoing validation. The original KSS score had several limitations including high variability, acknowledged by the developer and addressed by complete revision in 2011. When our study was designed, the original KSS had been the standard of use in our clinic and the new score was not yet available free of charge. This may justify the very weak correlation found in our study between the KSS patient form and function and all other tested scores. Knee OA is the common endpoint for a multitude of pathologies. Until present, there are no disease modifying drugs and treatment of early stages is mainly symptomatic. Total knee arthroplasty has become the mainstay for advanced disease in the elderly for many years, yet still some patients exhibit unreliable improvements. Prediction models may be one way to stratify patients at risk of poor outcomes. A group of researchers found low Oxford knee scores, poverty, increased body mass index, anxiety and depression to predict worse outcomes. In addition, there are also local factors such as impaired physical status and previous knee arthroscopy that are negative predictors of outcome. Contrarily, a fixed flexion deformity and absence of the anterior cruciate ligament were associated with postoperative improvement [18]. Machine learning algorithms are still at the beginning but show promising ability to predict which patients will achieve increased improvement after knee replacement [19].

\section{Conclusions}

The Romanian Knee disability and Osteoarthritis Outcome Score for Joint Replacement (KOOSJR) is a valid, reliable, consistent and reproducible clinical score for patients with OA requiring arthroplasty.

\section{Abbreviations}

OA (Osteoarthritis); PROs (Patient-reported outcomes); (KOOS) Knee injury and Osteoarthritis Outcome Score; KOOSJR (Knee disability and Osteoarthritis Outcome Score for Joint Replacement); ACR (American College of Rheumatology); EULAR (European League Against Rheumatism); IKDC (International Knee Documentation Committee - subjective knee form); KSS (knee society score - part1 knee score and part 2 function); EQ-5D-5L (Euroqol Index) and VAS (Euroqol visual analogue scale); ISPOR (International Society for Pharmacoeconomics and Outcomes Research); ICC (intraclass correlation coefficient); WOMAC (Western Ontario and McMaster Universities Osteoarthritis Index); PROMIS (Patient-Reported Outcomes Measurement Information System). 


\section{Declarations}

Acknowledgements: Deleanu Bogdan MD, PhD; Roxana Onofrei MD, PhD; loana Georgescu, MD; Andrei Ghiorghitoiu, MD; Roxana Forminte Med Stud.

Author's contributions: SF, HH and TA contributed to study conception and design. DV coordinated and managed all parts of the study. JMP carried out the literature search. HH conducted data collection and performed preliminary data preparations. BT conducted data analyses and all the authors contributed to the interpretation of data. SF wrote the first draft of the paper and all authors provided substantive feedback on the paper and contributed to the final manuscript.

Funding: No funding was obtained for this study.

Availability of data and materials: The datasets generated and/or analyzed during the current study are not publicly available but are available from the corresponding author on reasonable request.

Ethical approval and consent to participate. The study was approved by the Emergency clinical county hospital 'Pius Brinzeu' Timisoara 'Local ethics committee for scientific research'. All patients participated free-willingly and with written informed consent to the study.

Consent for publication: Not applicable.

Competing interests: The authors declare that they have no competing interests.

\section{References}

1. Martel-Pelletier J, Barr AJ, Cicuttini FM, et al. Osteoarthritis. Nat Rev Dis Primers. 2016 Oct 13;2:16072. doi: 10.1038/nrdp.2016.72.

2. Nguyen US, Zhang Y, Zhu Y, Niu J, Zhang B, Felson DT. Increasing prevalence of knee pain and symptomatic knee osteoarthritis: survey and cohort data. Ann Intern Med. 2011 Dec 6;155(11):72532. doi: 10.7326/0003-4819-155-11-201112060-00004.

3. Wilson I, Bohm E, Lübbeke A, et al. Orthopaedic registries with patient-reported outcome measures. EFORT Open Rev. 2019 3;4(6):357-367. doi: 10.1302/2058-5241.4.180080.

4. Rolfson O, Eresian Chenok K, Bohm E, et al Patient-reported outcome measures in arthroplasty registries. Acta Orthop. 2016 Jul;87 Suppl 1:3-8. doi:10.1080/17453674.2016.1181815.

5. Lyman S, Lee YY, Franklin PD et al. Validation of the KOOS, JR: a short-form knee arthroplasty outcomes survey. Clin Orthop Relat Res 2016;474:1461-71.

6. AAOS website https://www.aaos.org/uploadedFiles/PreProduction/Quality/Measures/IKDCEnglishUS.pdf (4 Sept 2019, date last accessed). 
7. Orthopaedicscores website https://www.orthopaedicscore.com/scorepages/knee_society_score.html (4 Sept 2019, date last accessed).

8. EuroQol website http://www.euroqol.org/about-eq-5d/how-to-use-eq-5d.html (4 Sept 2019, date last accessed).

9. Knee disability and Osteoarthritis Outcome Score (KOOS) website http://www.koos.nu/koosromanian.pdf (4 Sept 2019, date last accessed).

10. Onofrei RR, Amaricai E, Petroman R, Suciu O. Relative and absolute within-session reliability of the modified Star Excursion Balance Test in healthy elite athletes. PeerJ. 2019 Jun 12;7:e6999. doi: 10.7717/peerj.6999.

11. Lizaur-Utilla A, Miralles-Muñoz FA, Gonzalez-Parreño S, Lopez-Prats FA. Validation of the Spanish Version of the Knee Injury and Osteoarthritis Outcome Score (KOOS) for Elderly Patients With Total Knee Replacement. J Orthop Res. 2019 Jun 4. doi: 10.1002/jor.24386.

12. Multanen J, Honkanen M, Häkkinen A, Kiviranta I. Construct validity and reliability of the Finnish version of the Knee Injury and Osteoarthritis Outcome Score. BMC Musculoskelet Disord. 2018 May 22;19(1):155. doi: 10.1186/s12891-018-2078-7.

13. Huang CC, Chen WS, Tsai MW, Wang WT. Comparing the Chinese versions of two knee-specific questionnaires (IKDC and KOOS): reliability, validity, and responsiveness. Health Qual Life Outcomes. 2017 Dec 6;15(1):238. doi: 10.1186/s12955-017-0814-6.

14. Moutzouri M, Tsoumpos P, Billis E, Papoutsidakis A, Gliatis J. Cross-cultural translation and validation of the Greek version of the Knee Injury and Osteoarthritis Outcome Score (KOOS) in patients with total knee replacement. Disabil Rehabil. 2015;37(16):1477-83. doi: 10.3109/09638288.2014.972583.

15. Gandek B, Roos EM, Franklin PD, Ware JE Jr. A 12-item short form of the Knee injury and Osteoarthritis Outcome Score (KOOS-12): tests of reliability, validity and responsiveness. Osteoarthritis Cartilage. 2019 May;27(5):762-770. doi: 10.1016/j.joca.2019.01.011.

16. Austin DC, Torchia MT, Werth PM, Lucas AP, Moschetti WE, Jevsevar DS. A One-Question PatientReported Outcome Measure Is Comparable to Multiple-Question Measures in Total Knee Arthroplasty Patients. J Arthroplasty. 2019 Jul 24. pii: S0883-5403(19)30707-7. doi: 10.1016/j.arth.2019.07.023.

17. Hung $M$, Saltzman $C L$, Greene $T$ et al. Evaluating instrument responsiveness in joint function: the HOOS JR, the KOOS JR, and the PROMIS PF CAT. J Orthop Res 2017. doi:10.1002/jor.23739.

18. Sanchez-Santos MT, Garriga C, Judge A, et al. Development and validation of a clinical prediction model for patient-reported pain and function after primary total knee replacement surgery. Sci Rep. 2018 Feb 21;8(1):3381. doi: 10.1038/s41598-018-21714-1.

19. Fontana MA, Lyman S, Sarker GK, Padgett DE, MacLean CH. Can Machine Learning Algorithms Predict Which Patients Will Achieve Minimally Clinically Important Differences From Total Joint Arthroplasty? Clin Orthop Relat Res. 2019 Jun;477(6):1267-1279. doi: 10.1097/CORR.0000000000000687. 


\section{Tables}

Table.1 Spearman's rho correlation coefficients between the tested scores

\begin{tabular}{|c|c|c|c|c|c|c|c|}
\hline & KOOSJR1 & KOOSJR2 & Index & VAS & KSS1 & KSS2 & IKDC \\
\hline \multirow{3}{*}{ KOOSJR1 } & 1.000 & $.618^{* *}$ & $-.431^{* *}$ & $-.364^{* *}$ & -.133 & -.072 & $-.671^{* *}$ \\
\hline & & .000 & .000 & .000 & .192 & .485 & .000 \\
\hline & 100 & 45 & 100 & 99 & 98 & 97 & 99 \\
\hline \multirow{3}{*}{ KOOSJR2 } & $.618^{* *}$ & 1.000 & $-.563^{* *}$ & $-.438^{* *}$ & .020 & .131 & $-.484^{* * *}$ \\
\hline & .000 & & .000 & .003 & .898 & .402 & .001 \\
\hline & 45 & 46 & 45 & 45 & 44 & 43 & 45 \\
\hline \multirow{3}{*}{ Index } & $-.431^{* *}$ & $-.563^{* *}$ & 1.000 & $.556^{* *}$ & .100 & $.255^{*}$ & $.370^{* *}$ \\
\hline & .000 & .000 & & .000 & .330 & .012 & .000 \\
\hline & 100 & 45 & 100 & 99 & 98 & 97 & 99 \\
\hline \multirow{3}{*}{ VAS } & $-.364^{* *}$ & $-.438^{* *}$ & $.556^{* *}$ & 1.000 & $.229^{*}$ & $.249^{*}$ & $.377^{* *}$ \\
\hline & .000 & .003 & .000 & . & .024 & .014 & .000 \\
\hline & 99 & 45 & 99 & 99 & 97 & 96 & 98 \\
\hline \multirow{3}{*}{ KSS1 } & \begin{tabular}{|l|}
-.133 \\
\end{tabular} & .020 & .100 & $.229^{*}$ & 1.000 & $.359^{* *}$ & \begin{tabular}{|l|}
.129 \\
\end{tabular} \\
\hline & .192 & .898 & .330 & .024 & & .000 & .209 \\
\hline & 98 & 44 & 98 & 97 & 98 & 97 & 97 \\
\hline \multirow{3}{*}{ KSS2 } & -.072 & .131 & $.255^{*}$ & $.249^{*}$ & $.359^{* *}$ & 1.000 & .078 \\
\hline & \begin{tabular}{|l|}
.485 \\
\end{tabular} & .402 & .012 & .014 & .000 & & .448 \\
\hline & 97 & 43 & 97 & 96 & 97 & 97 & 96 \\
\hline \multirow{3}{*}{ IKDC } & $-.671^{* *}$ & $-.484^{* *}$ & $.370^{* *}$ & $.377^{* *}$ & .129 & .078 & 1.000 \\
\hline & .000 & .001 & .000 & .000 & .209 & .448 & \\
\hline & 99 & 45 & 99 & 98 & 97 & 96 & 99 \\
\hline
\end{tabular}

Figures 


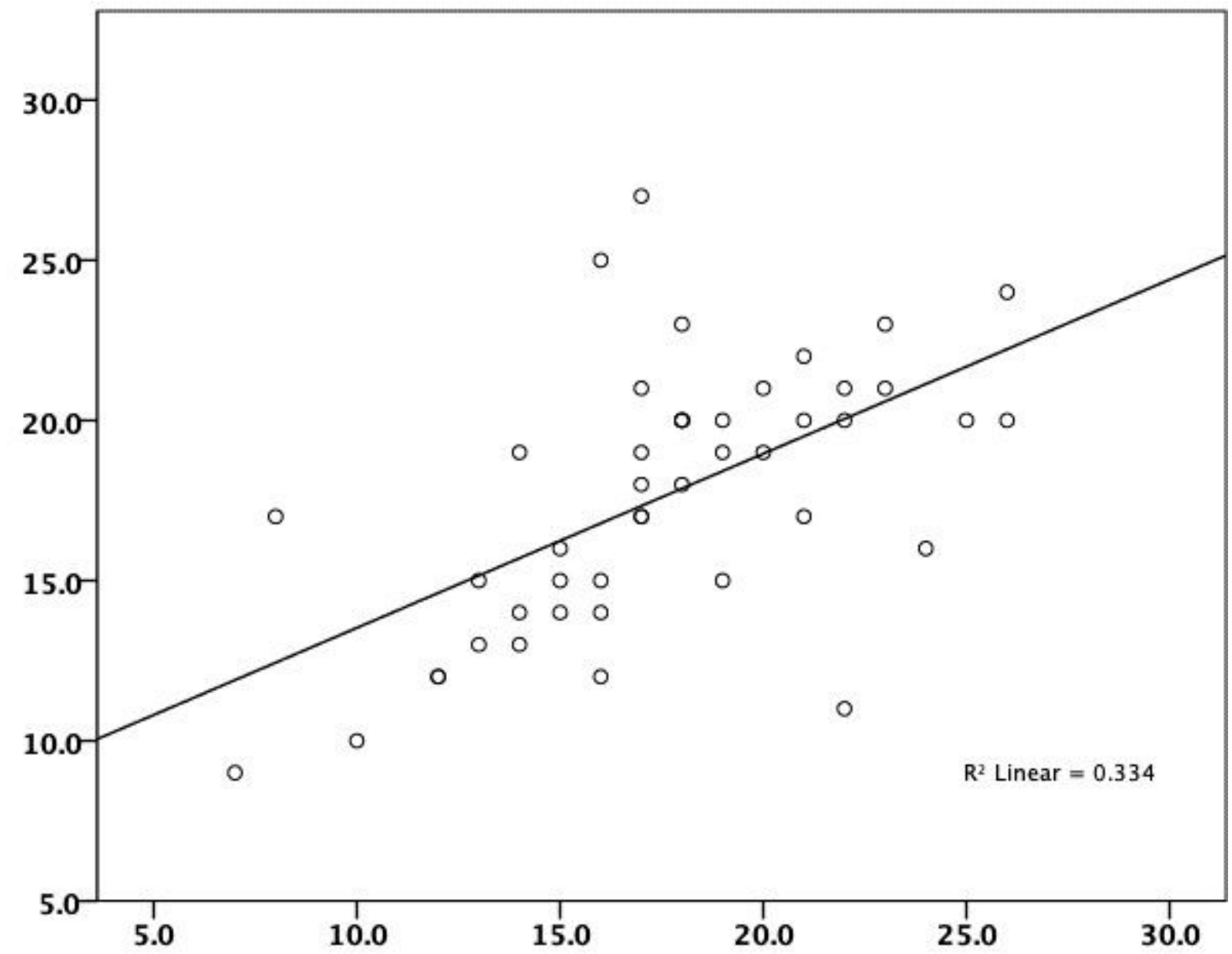

Figure 1

Moderate correlation between the first and repeated administration of the KOOSJR (Knee disability and Osteoarthritis Outcome Score for Joint Replacement) 


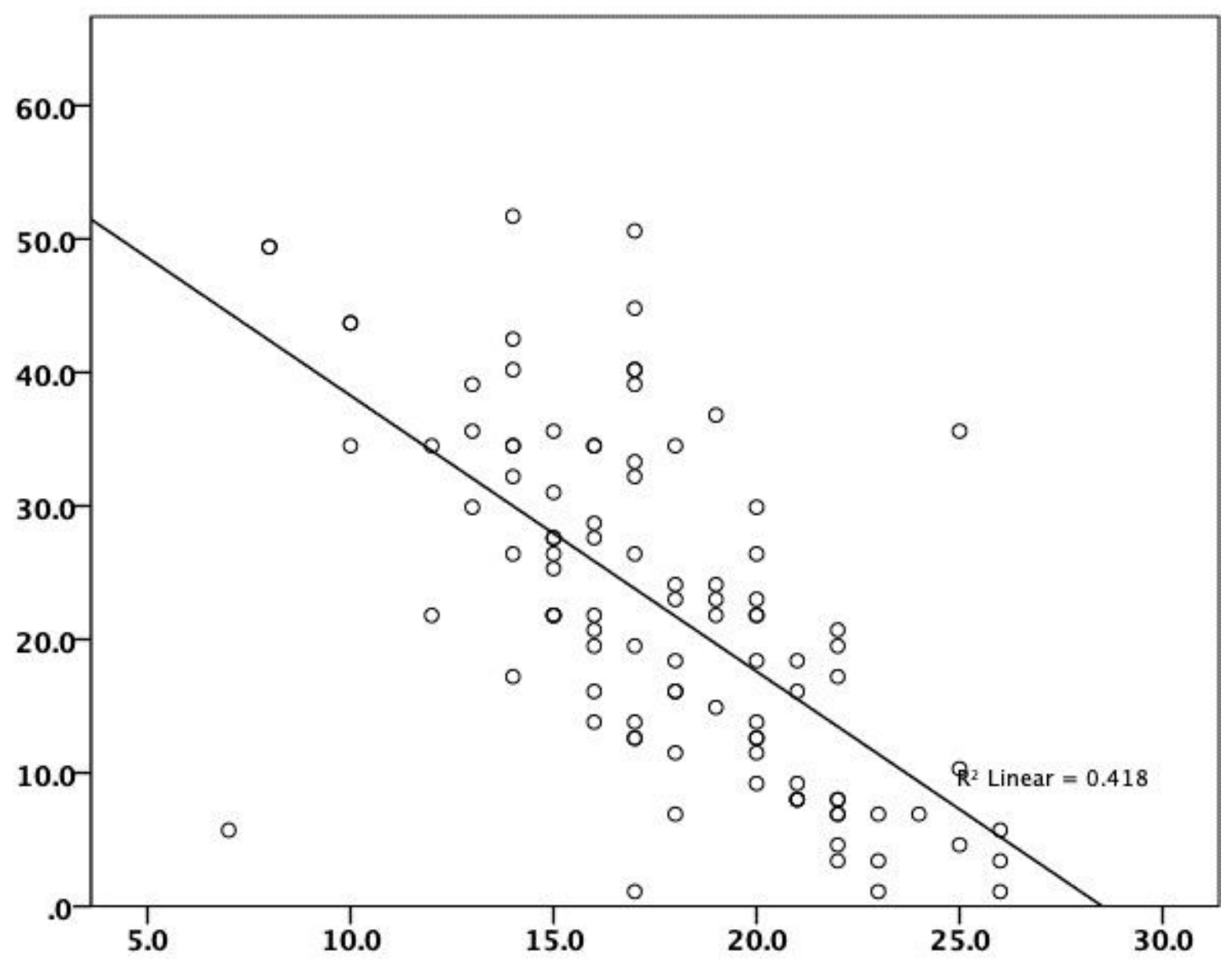

Figure 2

Moderate correlation between KOOSJR (Knee disability and Osteoarthritis Outcome Score for Joint Replacement) and IKDC (International Knee Documentation Committee - subjective knee form).

\section{Supplementary Files}

This is a list of supplementary files associated with this preprint. Click to download.

- KOOSJRrawJun2019.xIsx 